$15 \%, 218 ; 1968$ ) have now shown that rifamycin also inhibits stage two, the formation of the initiation complex. Rifamycin added to a complete system of enzyme, DNA and nucleoside triphosphate did not inhibit the incorporation of ${ }^{14} \mathrm{C}$ from ${ }^{14} \mathrm{C}$-cytidine triphosphate into nucleic acid, but when the enzyme was incubated with rifamycin before starting the reaction there was a very marked inhibition. Pre-incubation of the enzyme with DNA alone or GTP + ATP alone did not prevent the inhibition, but when all the components necessary for formation of the initiation complex (enzyme + DNA + ATP + GTP) were pre-incubated for only three minutes, the inhibitory effect of rifampicin was considerably reduced.

In the same issue of Biochimica Biophysica Acta $\left(15^{r y}, 215 ; 1968\right)$ Wehrli, Nüesch, Knüsel and Staehelin show that rifamycin SV or rifampicin are 10-100 times more potent inhibitors of bacterial RNA polymerase than actinomycin $C$, olivomycin or cinerubins $A$ and $B$. When tested against the RNA polymerase from rats, however, rifamycin was much less active than any of the other antibiotics, showing very little inhibition even at high concentrations. They showed that the inhibition caused by rifamycin depended on the enzyme concentration, whereas the inhibition caused by actinomycin did not, and suggested that rifamycin interac $\lrcorner$ s with the enzyme protein rather than the DNA primer. The difference in sensitivity of the bacterial and mammalian RNA polymerases was also found in the work with streptovaricin. This new work confirms that these enzymes must differ in the way in which RNA synthesis is initiated.

Further work on the inhibition of RNA polymerase by streptovaricin (Mizuno, Yamazaki, Knitta and Umezawa, Biochim. Biophys. Acta, 15\%, 322; 1968) has shown that streptovaricin has no effect on the enzyme from ascites tumour even at $20 \mu \mathrm{g}$ per $\mathrm{ml}$. when the $E$. coli enzyme is 85 per cent inhibited. After incubation with the mammalian polymerase there was no decrease in antibacterial action or the ability to inhibit bacterial RNA polymerase, which showed that the streptovaricin was not bound or inactivated by other proteins in the ascites tumour enzyme preparation. Kinetic studies showed that streptovaricin did not compete with DNA primer or CTP for binding sites on the bacterial enzyme. No binding of streptovaricin to DNA could be detected by difference spectrum, thermal transition curves, the antagonism of antibacterial action or sucrose gradient centrifugation with tritiated streptovaricin.

\section{Serum Proteins and Rejection}

Drs Robert E. Riggio, Gabriel H. Schwartz, Kurt H. Stenzel and Albert L. Rubin from the Rogosin Laboratories, Department of Biochemistry, New York Hospital - Cornell Medical Centre, have detected a protein in the sera of certain kidney graft recipients which bears a temporal relationship to the rejection state. So far, however, it is unknown whether this protein is specific to the process of rejection, or whether it merely reflects the inflammation and break-down effect associated with rejection.

According to the article in the current issue of Lancet, the protein in question is an a2-globulin. During the period that the investigation was carried out, raised levels of the protein seemed to point to unfavourable events in the survival of the graft, while normal levels indicated that a homograft rejection was unlikely. The research workers point out that several alterations in serum proteins have been observed after transplantation of organs in man and various tissues in animals for several years. While the nature of the changes remains unclear, their significanceif they are indeed related to the homograft rejectionmay lie in their ability to reflect the degree of therapeutically induced tolerance.

The results are based on data obtained from twentysix patients who received renal transplants at the New York Hospital between May 5, 1963, and October 10, 1967. Of these, twenty-five received a single renal transplant. Seventeen of the patients experienced a total of twenty-eight rejection episodes. When "rejection serum" was compared with serum obtained from recipients whose renal function was considered normal, the most consistent change was a quantitative increase in the a2-globulin fraction- $1,152 \mathrm{mg} / 100 \mathrm{ml}$. compared with $727 \mathrm{mg} / 100 \mathrm{ml}$. In addition, serumalbumin seemed to be reciprocally lowered during the same period so that total protein remained at a constant level of $6 \cdot 20 \mathrm{~g} / 100 \mathrm{ml}$. before rejection and $6.26 \mathrm{~g} / 100 \mathrm{ml}$. in mid-rejection. Seventeen of the episodes were characterized by an increase of 50 per cent or more in the $\alpha 2$-fraction during some phase of rejection; this increase did not appear until there was some indication of altered renal function. When graft rejection was aborted, in seventeen episodes the elevated a2-globulin returned to a lower or prerejection level. In six episodes, if the rejection process became chronic in course the fraction usually remained elevated as long as the graft remained in situ. When a rejected kidney was removed, however (two episodes), the elevated a2-globulin level returned to a lower or pre-rejection level.

The constant appearance of this protein with a homograft rejection and its virtual absence in almost all other conditions raise the possibility that this substance (or substances) may be specific to some phase of a rejection and/or tolerance process. The authors point out, however, that evidence of such a causal relationship is by indirect means only. Until the particular a2-globulin is identified, this finding cannot really be regarded as highly significant.

\section{Primary and Tertiary Structures}

\section{from our Molecular Biology Correspondent}

Somw interesting comparisons of sequences and tertiary structures in proteolytic enzymes have just become available. E. L. Smith and his group have determined the sequences of two subtilisins, proteases derived from different strains of the same species (B. subtilis). The first of these was reported last year, and belonged to the so-called $\mathrm{BPN}^{\prime}$ strain, and a series of five papers in J. Biol. Chem. (243, 2134 et seq.) now describe the determination of the sequence of the Carlsberg strain (the original subtilisin $A$ ). The two proteins show some small differences in their kinetic parameters, but are astonishingly different in sequence. Apart from one deletion in subtilisin Carlsberg, there are no fewer than 84 differences of sequence in the 275 residues. Of these, 61 correspond to single-base and 23 to double-base mutations, the proportion of the latter being of the order of the statistically expected 